\title{
Evaluación del índice aórtico abdominal y su relación con ruptura aneurismática abdominal en el Hospital de Especialidades del Centro Médico Nacional Siglo XXI
}

\section{Evaluation of the abdominal aortic index and its relationship with abdominal aneurysmal rupture at the Hospital de Especialidades, Centro Médico Nacional Siglo XXI}

\author{
Iván Velázquez del Valle-Lucero*, Jesús I. García-Palafox, José de J. García-Pérez y \\ Mayela Hernández-Juárez \\ Servicio de Angiología y Cirugía Vascular, Hospital de Especialidades, Centro Médico Nacional Siglo XXI, Ciudad de México, México
}

\begin{abstract}
Resumen
Objetivo: Determinar la relación entre índice aórtico (IA) y la ruptura de aneurisma de aorta abdominal (AAA) en una población mexicana del servicio de angiología del Centro Médico Nacional Siglo XXI. Métodos: Estudio retrospectivo en expedientes de pacientes operados de $A A A$, con variables como edad, género, índice tabáquico, comorbilidades, IA, diámetro aórtico máximo (DAM), perioperatorios, ruptura y mortalidad. El IA se calculó mediante somatometría y el DAM por angiotomografía y se empleó una descripción demográfica de población con análisis entre IA y ruptura. Resultados: Se estudiaron 93 casos (hombres, 76.3\%), con tabaquismo (80.6\%), hipertensión arterial sistémica (60.2\%) y operación abdominal previa (75.2\%) como comorbilidades más frecuentes. Se identificaron procedimientos de urgencia en $34.4 \%$ con mortalidad general a 24 horas posoperatorias de $11.8 \%$. Hasta $31.2 \%$ tuvo diagnóstico de aneurisma roto con DAM promedio de $69 \mathrm{~mm}$. Las complicaciones posquirúrgicas fueron respiratorias, isquémicas y renales con 30.1\%, 23.7\% y $9.7 \%$, respectivamente, $y$ se registró una estancia $<14$ días en $77.4 \%$. El IA tuvo diferencia estadísticamente significativa $(p<0.001$, IC95\%) entre grupos con y sin ruptura; se determinó un $I A \geq 4$ con razón de momios de 4.8 para ruptura de $A A A$, con sensibilidad y especificidad del $71.8 \%$ y $65.5 \%$. Conclusión: Un índice aórtico $\geq 4$ posee un riesgo mayor de ruptura en $A A A$, Io cual lo convierte en un parámetro predictor aceptable.
\end{abstract}

Palabras clave: Aorta. Diámetro. Índice. Ruptura. Mortalidad.

\begin{abstract}
Objective: To determine relationship between Aortic Index (IA) and ruptured Abdominal Aortic Aneurysm (AAA) in Mexican population from the Angiology Service, Centro Médico Nacional Siglo XXI. Methods: Retrospective study in records of patients operated for $A A A$, with variables such as age, gender, smoking index, comorbidities, Al, maximum aortic diameter (MAD), perioperative, rupture and mortality. The Al was calculated with somatometry and MAD by angiotomography; and demographic description of population with analysis between Al and rupture. Results: 93 cases (76.3\% men) with smoking (80.6\%), high
\end{abstract}

Correspondencia:

*Iván Velázquez del Valle-Lucero

E-mail: xitlaian@gmail.com
Available online: $15-04-2021$ Rev Mex Angiol. 2021;49(1):1-7 www.RMAngiologia.com 0377-4740/@ 2021 Sociedad Mexicana de Angiología y Cirugía Vascular y Endovascular, A.C. Publicado por Permanyer. Este es un artículo open access bajo la licencia CC BY-NC-ND license (http://creativecommons.org/licenses/by-nc-nd/4.0/). 
blood pressure (60.2\%) and previous abdominal surgery $(75.2 \%)$ were studied as the most frequent comorbidities. Emergency procedures in $34.4 \%$ with general mortality at 24 hours postoperatively of $11.8 \% .31 .2 \%$ presented diagnosis of ruptured aneurysm with an average MAD of $69 \mathrm{~mm}$. Post-surgical complications were respiratory, ischemic and renal with 30.1, 23.7 and $9.7 \%$ respectively; and a stay $<14$ days in $77.4 \%$. The Al presented statistically significant difference $(p<0.001,95 \% \mathrm{Cl})$ between groups with and without rupture; determining an $A l \geq 4$ with odds ratio of 4.8 for AAA rupture, with sensitivity and specificity of 71.8 and $65.5 \%$. Conclusion: An aortic index $\geq 4$ has a higher risk of rupture in $A A A$, establishing it as an acceptable predictor parameter.

Key words: Aorta. Diameter. Index. Rupture. Mortality.

\section{Introducción}

El aneurisma aórtico abdominal (AAA) se define como la dilatación de la aorta abdominal $>3 \mathrm{~cm}$ en su corte axial o $50 \%$ mayor al diámetro contiguo sano; es una anomalía de efectos potencialmente letales y el principal de ellos su ruptura ${ }^{1}$.

La aorta abdominal infrarrenal es el sitio de mayor afectación con el $30 \%$ de los casos y es consecuencia, entre otros factores, de una menor densidad de unidades laminares en la pared arterial; se encuentra en el $1 \%$ de la población de 55 a 64 años de edad, e incrementa su prevalencia de $2 \%$ a $4 \%$ por cada década, con más frecuencia en hombres con una relación de 4:1. Se ha documentado la predisposición en varones por su mayor diámetro aórtico sin aneurisma y se considera un factor de riesgo independiente ${ }^{2,3}$.

Su diagnóstico se establece por estudios de imagen en pacientes con factores de riesgo o como hallazgo incidental en protocolos de estudio por otra enfermedad. Para el estudio de la morfología, composición y dimensiones del aneurisma es necesario una angiotomografía o resonancia magnética; la primera tiene la ventaja de mostrar placas calcificadas de la pared aórtica que define decisiones terapéuticas ${ }^{4,5}$.

La ruptura es la complicación de mayor mortalidad con $86 \%$ a $89 \%$ y es proporcional al diámetro aneurismático. Durante el posoperatorio, las complicaciones son más comunes en los procedimientos abiertos electivos que exigen mayor estancia hospitalaria, como la neumonía, incluso con mayor mortalidad (5\%-8\%), en contraste con el 3.5\% del tratamiento endovascular. Múltiples estudios incluyen el costo de estas complicaciones, pero ambos tratamientos se equilibran con un aproximado de 40,000 dólares (en el tratamiento endovascular, la endoprótesis representa el costo mayor final). El contraste se refleja en la calidad de vida; el mejor tratamiento es el endovascular en virtud de su menor tasa de complicaciones ${ }^{6}$. En cambio, para la reparación abierta del aneurisma abdominal roto se calcula un costo promedio de 28,000 dólares tan sólo en el internamiento inicial, sin considerar tratamiento y seguimiento de las complicaciones posoperatorias y su elevada mortalidad perioperatoria?.

Se ha estudiado la previsión de una ruptura aneurismática en las últimas décadas, pero el diámetro aórtico máximo $(5-5.5 \mathrm{~cm})$ es aún el predictor más sólido de ruptura ${ }^{1}$. Algunos estudios describen los factores etiológicos, entre ellos la diferencia en diámetro del cuello del aneurisma, calcificación, volumen del trombo intraluminal y su presencia parcial o circunferencial, volumen total del aneurisma, longitud de arterias iliacas e índice aórti$\mathrm{co}$; todos han tenido resultados significativos en aneurismas rotos e intactos con dimensiones similares ${ }^{8,9}$.

El índice aórtico (IA) es un valor numérico que expresa la relación entre diámetro máximo aneurismático del paciente $(\mathrm{cm})$ y su superficie corporal $\left(\mathrm{m}^{2}\right)$, y debe cuantificarse como dato de utilidad para el diagnóstico, la decisión terapéutica y la ruptura de esta entidad. Aún no se establece la cifra de corte del IA para definir un aneurisma; sin embargo, hasta el momento sólo se considera 1.65 como la cifra equivalente a $3 \mathrm{~cm}$ de diámetro (tamaño conceptual) en poblaciones estudiadas de hombres y es una referencia inicial para estudios de prevalencia ${ }^{10}$. El IA, pese a que aún no es una medida estandarizada, es el mejor predictor de riesgo de ruptura al adaptarse con precisión a la complexión corporal de la población femenina, en comparación con la definición absoluta de aneurisma, que se limita sólo a cifras del diámetro aneurismático ${ }^{6}$.

Ante los costos de una reparación quirúrgica de urgencia en el AAA y su tasa elevada de mortalidad por ruptura, el objetivo de este estudio es determinar la relación entre el índice aórtico y la ruptura del AAA en una población que requirió intervención quirúrgica electiva y de urgencia en el servicio de cirugía vascular del Centro Médico Nacional Siglo XXI entre los años 2015 y 2019.

\section{Métodos}

Se realizó un estudio de tipo retrospectivo y se recogió la información de los expedientes clínicos de los 
pacientes sometidos a intervenciones por aneurisma de aorta abdominal infrarrenal en el servicio de angiología y cirugía vascular del Hospital de Especialidades del Centro Médico Nacional Siglo XXI, en el periodo del 1 de enero de 2015 al 31 de diciembre del 2019.

Se incluyó a pacientes de ambos sexos mayores de 18 años sometidos a operaciones abiertas de aorta abdominal electiva y de urgencia, sin incluir a los pacientes con afectación aórtica de causa infecciosa o inflamatoria, procedimiento aórtico previo o sin registro de datos perioperatorios o somatometría para determinación del índice aórtico. Con un total de 93 pacientes para el análisis final, se registraron variables como edad, género, superficie corporal, índice tabáquico, comorbilidades, complicaciones posoperatorias, índice aórtico, ruptura y mortalidad.

El índice aórtico se calculó como variable independiente mediante el peso, superficie corporal y diámetro aórtico máximo medido por angiotomografía en todos los casos. Con las variables de interés se efectuó una descripción demográfica y de comorbilidades de la población de estudio, además de una búsqueda de relación con el índice aórtico y la relación directa con ruptura y mortalidad.

Se vincularon en particular la ruptura de aneurisma, la mortalidad y las complicaciones en las operaciones electivas y de urgencia; el objetivo final fue comprobar o desestimar la hipótesis y obtener resultados secundarios de interés clínico predictivo. Las variables de interés se concentraron en una base de datos (Excel, Microsoft) y una vez codificadas se llevó a cabo un análisis estadístico mediante el programa STATA 13 (StataCorp). Se obtuvo la descripción de variables y análisis estratificado con base en la ruptura abdominal y se expresaron en porcentajes, medias y desviación estándar de acuerdo con la variable; este análisis se realizó con las pruebas estadísticas ji cuadrada, exacta de Fisher, $t$ de Student y Wilcoxon-Mann-Whitney según fuera el tipo de variable. En una segunda fase se analizó la distribución del índice aórtico entre dos grupos con diferencias estadísticamente significativas $(p<0.05)$ y se fijó un punto de corte, del cual se efectuó un análisis bivariado entre índice aórtico (medición preoperatoria) y ruptura con la finalidad de determinar una relación y luego calcular sensibilidad, especificidad y poder predictivo junto con un gráfico de área bajo la curva (curva ROC).

\section{Resultados}

En la base de datos interna se identificaron 118 casos para revisión y análisis de investigación de acuerdo con
Tabla 1. Variables preoperatorias y comorbilidades, n $(\%)$

\begin{tabular}{|l|c|}
\hline Hombres & $71(76.3)$ \\
\hline Edad, media (desviación estándar) & $69.5(34.5)$ \\
\hline $\begin{array}{l}\text { Índice de masa corporal, media (desviación } \\
\text { estándar) }\end{array}$ & $25.4(10.8)$ \\
\hline Obesidad & $13(13.9)$ \\
\hline Bajo peso & $8(8.6)$ \\
\hline Tabaquismo & $75(80.6)$ \\
\hline Hábito tabáquico actual & $14(15.1)$ \\
\hline Hábito tabáquico suspendido & $61(65.6)$ \\
\hline Sin hábito tabáquico & $18(19.3)$ \\
\hline Índice tabáquico, mediana (RIC)* & $25.1(15.9-34.5)$ \\
\hline Diabetes mellitus de tipo 2 & $16(17.2)$ \\
\hline Hipertensión arterial sistémica & $56(60.2)$ \\
\hline Dislipidemia & $22(23.6)$ \\
\hline Cardiopatía isquémica & $19(20.4)$ \\
\hline Insuficiencia renal crónica & $9(9.7)$ \\
\hline Neumopatía crónica & $11(11.8)$ \\
\hline Episodio vascular cerebral & $1(1.1)$ \\
\hline Arritmias cardiacas & $2(2.2)$ \\
\hline Operación abdominal & $70(75.2)$ \\
\hline Alcoholismo & $35(37.6)$ \\
\hline Rano & \\
\hline
\end{tabular}

*Rango intercuartílico.

los criterios de inclusión; esta cifra se redujo a 93 pacientes después de aplicar los criterios de exclusión. De los casos analizados, los datos demográficos se simplifican en la Tabla 1. Los promedios en peso y talla fueron de $69.1 \mathrm{~kg}( \pm 37)$ y $1.64 \mathrm{~m}( \pm 0.19)$, respectivamente, con un índice de masa corporal de 25.41 ( \pm 10.83) promedio; se detectó a $13(13.9 \%)$ pacientes en estado de obesidad y $8(8.6 \%)$ con peso bajo.

El tabaquismo como principal factor de riesgo se encontró como antecedente en el $80.6 \%$ de la población y de este grupo 14 pacientes (15.05\%) todavía mantenían el hábito tabáquico al momento del procedimiento. Por otra parte, el índice tabáquico registró un promedio de 25.1 paquetes/año con un rango intercuartílico $(\mathrm{RIC})$ de 15.9 a 34.5 en la población general, y un promedio en los fumadores activos de 27.6 paquetes/año; sólo el 19.3\% ( $n=18)$ no tenía antecedente de tabaquismo. Con respecto a las comorbilidades de 
Tabla 2. Variables transoperatorias, n (\%)

\begin{tabular}{|l|c|}
\hline $\begin{array}{l}\text { Diagnóstico preoperatorio } \\
\text { Aneurisma de aorta abdominal roto } \\
\text { Aneurisma de aorta abdominal no roto }\end{array}$ & $29(31.2)$ \\
\hline $\begin{array}{l}\text { Aneurisma de aorta abdominal (supra/yuxta) } \\
\text { AAA + otros (insuficiencia, trombosis, etc.) }\end{array}$ & $7(78.5)$ \\
\hline $\begin{array}{l}\text { Carácter del procedimiento } \\
\text { Electivo } \\
\text { Urgente }\end{array}$ & $5(5.4)$ \\
\hline Afectación de arterias ilíacas & $61(65.6)$ \\
\hline $\begin{array}{l}\text { Derivación } \\
\text { Aortoilíaco } \\
\text { Aortofemoral } \\
\text { No especificado }\end{array}$ & $32(34.4)$ \\
\hline Exploración vascular distal & $23(24.7)$ \\
\hline Mortalidad transoperatoria $0<24 \mathrm{~h}$ & $65(69.9)$ \\
\hline
\end{tabular}

la población, se encontró con mayor prevalencia la hipertensión arterial sistémica y la operación abdominal; las comorbilidades menos frecuentes fueron la insuficiencia renal crónica, los episodios vasculares cerebrales y las arritmias cardiacas. Los datos y sucesos transoperatorios obtenidos se describen en la Tabla 2, en la que puede observarse que un tercio de los procedimientos tuvo carácter urgente y de éstos más del $90 \%$ se debió a un aneurisma roto.

En mortalidad transoperatoria y en las primeras 24 horas posoperatorias se registraron cifras más altas a lo informado en las publicaciones médicas; sin embargo, de las 11 muertes ocurridas, todas correspondieron a intervenciones de carácter urgente con el diagnóstico de aneurisma roto anterior $(n=10)$ o trombosis $(n=1)$.

En variables cuantificables por imagen se observó un diámetro aórtico máximo promedio de $69 \mathrm{~mm}$, cercano a la cifra de corte de $70 \mathrm{~mm}$ que implica un riesgo de ruptura $>30 \%$ anual. Esto señala que en la unidad se practica un procedimiento quirúrgico abierto en estadios avanzados de la enfermedad, lo que supone problemas en el diagnóstico temprano, protocolos o seguimiento, factores que no son de interés en este estudio.

Las complicaciones posquirúrgicas más frecuentes fueron isquemia, insuficiencia renal y complicaciones respiratorias, estas últimas presentes en la mitad de la población. Sólo en $39.8 \%$ no se presentaron complicaciones y la cifra en los sometidos a operación electiva fue de $36.1 \%$. En los pacientes complicados, el $56.3 \%$ $(n=9)$ se vinculó con obesidad y estancia prolongada (> $10 \mathrm{EIH}$ ), que son factores de riesgo ampliamente

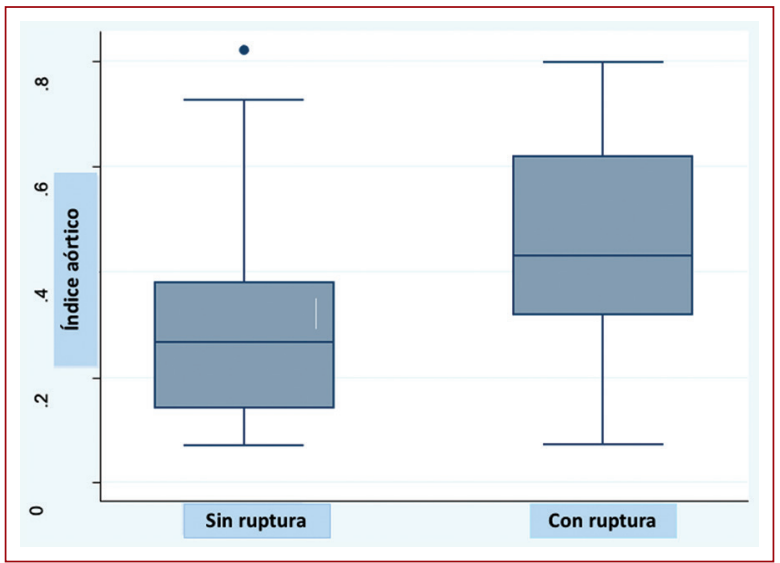

Figura 1. Gráfica de cajas que muestra la media del índice aórtico por grupo con los percentiles $25^{\circ} / 75^{\circ}$.

descritos. También se identificó el escenario de múltiples complicaciones con $23.6 \%(n=22)$ de los casos y de ellos un tercio $(n=7)$ tuvo una mortalidad temprana posoperatoria, lo que constituye un factor predictor de mal pronóstico.

La estancia intrahospitalaria, sin considerar a los pacientes con mortalidad temprana $(<30$ días), tuvo un promedio de 7.95 días con un rango intercuartílico de 6 a 10 días y una mediana de 9 días. El $77.4 \%$ de los pacientes tuvo una estancia hospitalaria menor de 14 días (Tabla 3). La mortalidad a 30 días hallada en la población general y en operaciones de urgencia se mantuvo en cifras de estudios amplios de mortalidad, aunque en los procedimientos electivos muestra un ligero aumento.

En el análisis de variables de interés en la población estratificada por ruptura de aneurisma de aorta abdominal no se reconocieron diferencias estadísticamente significativas en relación con características de la población, comorbilidades e índices y puntuaciones de ruptura bajo las pruebas estadísticas de ji cuadrada, exacta de Fisher, $t$ de Student y Wilcoxon-Mann-Whitney; no obstante, tanto el diámetro aórtico máximo como el índice aórtico en un análisis univariado ( $t$ de Student) revelaron diferencias estadísticamente significativas ( $p>0.0001)$ con un intervalo de confianza del 95\% (Tabla 4).

Ante este resultado, y con apoyo de la distribución del índice aórtico entre los dos grupos (Fig. 1), se fijó un punto de corte para el índice aórtico. De esta manera se determinó un índice aórtico $\geq 4$ con una razón de momios (odds ratio) de 4.86 para la ruptura de aneurisma de aorta abdominal, con un intervalo de confianza de $95 \%$ y una $p<0.001$. 
Tabla 3. Estancia intrahospitalaria (EIH) prolongada y mortalidad a 30 días, $\mathrm{n}(\%)$

\begin{tabular}{|l|c|}
\hline EIH $<7$ días & $26(27.9)$ \\
\hline EIH $7-14$ días & $46(49.5)$ \\
\hline EIH $>14$ días & $12(12.9)$ \\
\hline Mortalidad a 30 días & \\
Total $(n=93)$ & $27(29)$ \\
Operación electiva $(n=71)$ & $9(12.7)$ \\
\hline Urgencia $(n=32)$ & $18(56.2)$ \\
\hline
\end{tabular}

Tabla 4. Características basales de la población por ruptura de aneurisma abdominal $(n=93)$

\begin{tabular}{|c|c|c|c|}
\hline Variable & $\begin{array}{l}\text { Sin ruptura } \\
(n=61, \%)\end{array}$ & $\begin{array}{l}\text { Con ruptura } \\
(n=32, \%)\end{array}$ & Valor $\mathbf{p}$ \\
\hline $\begin{array}{l}\text { Diámetro máximo } \\
\text { de aneurisma } \\
(\mathrm{mm}) ; \text { media (SD) }\end{array}$ & $62.30(18.60)$ & $82.14(20.17)$ & 0.001 \\
\hline $\begin{array}{l}\text { Índice aórtico } \\
\text { abdominal; } \\
\text { media (SD) }\end{array}$ & 3.57 (1.12) & $4.62(1.18)$ & 0.001 \\
\hline \multicolumn{4}{|c|}{$\begin{array}{l}\text { Análisis univariado para ruptura de aneurisma abdominal } \\
\text { según el punto de corte del índice aórtico abdominal }\end{array}$} \\
\hline $\begin{array}{l}\text { Índice aórtico } \\
\text { abdominal }\end{array}$ & OR & IC95\% $\%$ & Valor $\mathbf{P}$ \\
\hline$\geq 4$ & 4.86 & $(1.91-12.38)$ & 0.001 \\
\hline
\end{tabular}

Ante este hallazgo estadístico se realizó un cálculo de sensibilidad $(71.8 \%)$, especificidad $(65.5 \%)$ y poder predictivo negativo (81.6\%) del índice aórtico para ruptura de aneurisma de aorta abdominal y se graficó mediante área bajo la curva (Fig. 2). Esto convierte al índice aórtico en una prueba de tamizaje de capacidad aceptable como predictor, si se considera que la ruptura es una complicación de elevada mortalidad en esta afección.

\section{Discusión}

Algunos estudios de cohorte amplios registran cifras heterogéneas entre la definición absoluta de aneurisma $(30 \mathrm{~mm})$ de aorta abdominal y la relativa ( $50 \%$ superior al diámetro aórtico sano), con amplios cambios en relación con la superficie corporal del individuo. Con el IA, la relación cambia al aumentar 1.5 veces en pacientes con baja superficie corporal y dimensiones aneurismáticas $<30 \mathrm{~mm}$, pero disminuye 1.5 veces en pacientes con superficie corporal alta y dimensiones

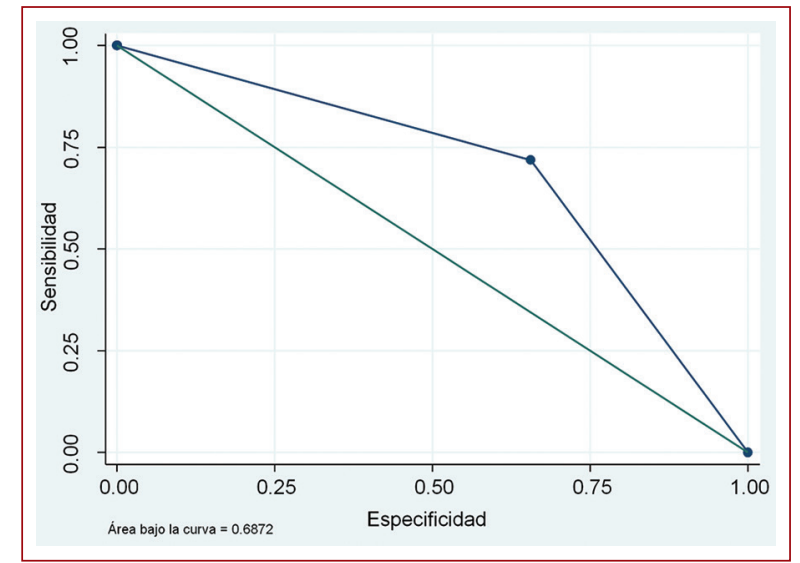

Figura 2. Área bajo la curva para un índice aórtico > 4 .

aneurismáticas > $30 \mathrm{~mm}^{6,11}$, situación que se presenta en este estudio. Starck, et al., concluyeron que los aneurismas $<30 \mathrm{~mm}$ son más grandes de lo previsto de acuerdo con la superficie corporal del paciente, lo que se confirma en los pacientes de este análisis con aneurisma $<5.5 \mathrm{~cm}$ que se manifiestan con ruptura. Jones, et al. muestran el punto anterior específico al grupo de mujeres con tendencia relativa a mostrar menores dimensiones de superficie corporal, con una prevalencia de aneurisma que aumenta al utilizar el índice aórtico respecto de las definiciones absolutas y relativas ya expuestas; no obstante, la prevalencia se mantiene en la población masculina. Nyronnig, et al., relacionan más el índice aórtico con crecimiento aórtico $>5 \mathrm{~mm}$ en comparación con el diámetro aórtico, y no como mejor predictor de ruptura; esta variable es importante para analizar, si bien exige una distinta metodología con variables de interés que cubran un seguimiento de estos pacientes por la consulta externa, datos no disponibles en este estudio. Por último, Lo, et al. definieron el diámetro aórtico como principal indicador de ruptura en hombres y el índice aórtico para las mujeres con marcada diferencia, aunque en este estudio se demuestra que no hay diferencia por género y que se manifiesta el riesgo de ruptura dependiente sólo del índice aórtico. La mayor parte de los estudios se ha conducido en una población europea, y también existen representaciones asiáticas, en Oceanía y Estados Unidos, pero ninguna en una población latinoamericana $a^{10-14}$.

En cuanto a este punto en particular, una población como la latinoamericana, o de modo específico la mexicana, tiende a ser un dato relevante, ya que esta disminución de las dimensiones de un aneurisma para su definición o ruptura de acuerdo con la superficie 
corporal podría colocar a esta población en la misma situación que el grupo de mujeres de otras regiones ya estudiadas, debido a la menor estatura promedio de la población latinoamericana. Lo anterior induce a introducir un gran cambio o reto en las definiciones del aneurisma de aorta abdominal, sus dimensiones como criterio diagnóstico y, aún más importante, la predicción de ruptura o cifras para decidir una reparación electiva.

La repercusión no sólo implicaría la reducción de la incidencia de ruptura de aneurisma de aorta abdominal y la indicación de reparación electiva antes de su complicación, sino también la valoración de la incidencia de complicaciones posquirúrgicas de acuerdo con el índice aórtico abdominal. Estudios como el de Deery, et al. han publicado mayor mortalidad y más complicaciones posquirúrgicas en los primeros 30 días posoperatorios en mujeres, tras atribuir la razón a la disparidad del índice aórtico frente a los hombres en el momento del procedimiento; en última instancia, el índice aórtico es relevante como parámetro en comparación con el diámetro aórtico, este último descartado como variable significativa en los resultados posoperatorios de otros análisis publicados ${ }^{15,16}$. En este estudio, lo anterior podría explicar el mayor número estadístico de complicaciones posoperatorias en la población respecto a lo notificado en las publicaciones actuales, lo cual siempre se ha atribuido a una detección más tardía y protocolos preoperatorios prolongados. Sin embargo, es un aspecto importante de valorar y exige investigación para encontrar una respuesta a la tasa de complicaciones y mortalidad en este país, con un claro índice mayor y un diámetro aórtico promedio al momento de la intervención $n^{17,18}$.

El potencial analítico para el índice aórtico abdominal es amplio y ya se ha determinado una repercusión en la evolución posoperatoria a largo plazo, como la mortalidad a un año. Un índice aórtico mayor al momento del procedimiento quirúrgico representa un mayor riesgo de complicaciones y a largo plazo se ha documentado mayor mortalidad en mujeres ${ }^{19}$. Dada la metodología de este estudio, no se analizó ni determinó la mortalidad a mediano plazo de acuerdo con el índice aórtico abdominal, pese a lo cual es una variable que todavía debe estudiarse, entre muchas otras investigaciones futuras.

\section{Conclusiones}

Los pacientes con un índice aórtico $\geq 4$ poseen un riesgo 4.8 veces mayor de sufrir ruptura de aneurisma de la aorta abdominal con una sensibilidad y especificidad mayores de $71.87 \%$ y $65.57 \%$, respectivamente; el índice aórtico es un parámetro de tamizaje aceptable como predictor de ruptura de aneurisma de aorta abdominal.

Este estudio no requirió carta de consentimiento informado puesto que es un estudio retrospectivo y los datos se obtuvieron de una base de datos interna del servicio de angiología y cirugía vascular y los expedientes clínicos físico y electrónico institucionales. El protocolo recibió aprobación de los comités de investigación y ética del hospital, con número de registro F-2020-3601-188.

\section{Agradecimientos}

A mis padres, Ian, Ale y Jessi; gracias a ustedes por estar a mi lado, apoyándome en esta etapa tan importante de mi vida.

\section{Financiamiento}

No se recibió financiamiento alguno para la conducción de este estudio.

\section{Conflicto de intereses}

Los autores declaran no tener ningún conflicto de intereses.

\section{Responsabilidades éticas}

Protección de personas y animales. Los autores declaran que para esta investigación no se han realizado experimentos en seres humanos ni en animales.

Confidencialidad de los datos. Los autores declaran que han seguido los protocolos de su centro de trabajo sobre la publicación de datos de pacientes.

Derecho a la privacidad y consentimiento informado. Los autores declaran que en este artículo no aparecen datos de pacientes.

\section{Bibliografía}

1. Carino D, Sarac T, Elefteriades J. Abdominal aortic aneurysm: evolving controversies and uncertainties. International Journal of Angiology. 2018;27(2):058-080

2. Tracci MC, Roy RA, Upchurch GR. Chapter 70. Aortoiliac aneurysms: evaluation, decision making, and medical management. Cronenwett $\mathrm{J}$. Wayne K. Rutherford's vascular surgery. 9th ed. EU: Elsevier, 2018;1:884-893

3. Solberg S, Forsdahl SH, Singh K, Jacobsen BK. Diameter of the infrarenal aorta as a risk factor for abdominal aortic aneurysm: The Tromsø Study, 1994-2001. European Journal of Vascular and Endovascular Surgery. 2010;39(3):280-284. 
4. Moll FL, Powell JT, Fraedrich G. Management of abdominal aortic aneurysms clinical practice guidelines of the European Society. European Journal Vascular and Endovascular Surgery. 2011;41(supp 1):S1eS58.

5. Public Health England. National Screening Committee: abdominal aortic aneurysm screening; 27 July 2015.

6. Chaikof E, Dalman R. Guía de práctica clínica de la Society for Vascular Surgery sobre la asistencia de los pacientes con aneurisma aórtico abdominal. Journal of Vascular Surgery 2018; Society for Vascular Surgery:1-84.

7. Patel ST. The cost-effectiveness of repairing ruptured abdominal aortic aneurysms. Journal of Vascular Surgery. 2000;32(2):247-257.

8. Spanos K, Nana P, Kouvelos G, Mpatzalexis K, Matsagkas M, Giannoukas AD. Anatomical differences between intact and ruptured large abdominal aortic aneurysms. Journal of Endovascular Therapy. 2020;27(1):117-123.

9. Lo RC, Bensley RP, Hamdan AD, Wyers M, Adams JE, Schermerhorn ML. Gender differences in abdominal aortic aneurysm presentation, repair, and mortality in the Vascular Study Group of New England. Journal of Vascular Surgery. 2013;57(5):1261-1268.e5.

10. Jones GT, Sandiford P, Hill GB, Williams MJA, Khashram M, Tilyard MW, van Rij AM. Correcting for body surface area identifies the true prevalence of abdominal aortic aneurysm in screened women. European Journal of Vascular and Endovascular Surgery. 2019;57(2):221-228.

11. Starck J, Aaltonen HL, Björses K, Lundgren F, Gottsäter A. A significan t correlation between body surface area and infrarenal aortic diameter is detected in a large screening population with possibly clinical implications. International Angiology. 2019:38(5):395-401
12. Lo RC, Lu B, Fokkema, Conrad M, Patel VI, Fillinger M, et al. Relative importance of aneurysm diameter and body size for predicting abdominal aortic aneurysm rupture in men and women. Journal of Vascular Surgery. 2014;59(5):1209-1216

13. Siika A. Novel markers of rupture in small abdominal aortic aneurysms: supra-renal aortic size index and peak wall rupture index. European Journal of Vascular and Endovascular Surgery. 58(6):e308.

14. Alldstedt L, Skoog P. Is the aortic size index relevant as a predictor of abdominal aortic aneurysm? A population-based prospective study: the Tromsø study. Original article. Scandinavian Cardiovascular Journal. 2020;54(2):130-13

15. Sahal M, Prusa AM, Wibmer A, Wolff KS, Lammer J, Polterauer P, et al. Elective abdominal aortic aneurysm repair: does the aneurysm diameter influence long-term survival? European Journal of Vascular and Endovascular Surgery. 2008:35(3):288-294.

16. Ramos C, Pujari A, Rajani RR, Escobar GA, Rubin BG, Jordan WD. et al. Perioperative outcomes for abdominal aortic aneurysm repair based on aneurysm diameter. Vascular and Endovascular Surgery. 2020;54(4):341-347.

17. Matyal R, Shakil O, Hess PE, Lo R, Jainandunsing JS, Mahmood B. Impact of gender and body surface area on outcome after abdominal aortic aneurysm repair. The American Journal of Surgery. 2015:209(2):315-323.

18. Deery SE, Soden PA, Zettervall SL, Shean KE, Bodewes TCF, Pothof AB. Sex differences in mortality and morbidity following repair of intact abdominal aortic aneurysms. Journal of Vascular Surgery. 2017;65(4):1006-1013.

19. Jones DW, Deery SE, Schneider DB, Rybin DV, Siracuse JJ, Farber A. Differences in patient selection and outcomes based on abdominal aortic aneurysm diameter thresholds in the vascular quality initiative. Journal of Vascular Surgery. 2019;70(5):1446-145 